\title{
El crecimiento económico como modelo de desarrollo social y su relación con el cambio climático
}

\section{Economic growth as a model of social development and its relation with the climate change}

Dr. Gabriel M. Rodríguez Pérez de Agreda es profesor titular de la Facultad Latinoamericana de Ciencias Sociales (FLACSO), Programa Cuba, Universidad de La Habana (gabriel@flacso.uh.cu) (http://orcid.org/0000-0002-7656-1774)

Dra. Elizabeth Cabalé Miranda es profesora titular de la Facultad Latinoamericana de Ciencias Sociales (FLACSO), Programa Cuba, Universidad de La Habana. (elicabalem@flacso.uh.cu) (http://orcid.org/0000-0002-4373-5221)

Dania Deroy Domínguez es profesora instructora de la Facultad Latinoamericana de Ciencias Sociales (FLACSO), Programa Cuba, Universidad de La Habana. (danita@flacso.uh.cu) (http://orcid.org/0000-0002-5156-7950)

\section{Resumen}

El presente artículo analiza cómo desde el pensamiento crítico se abordan las complejas relaciones entre crecimiento económico, como modelo de desarrollo social imperante, y el cambio climático. En este sentido, el cambio climático se presenta como el fenómeno global más gravitante al cual se enfrenta la humanidad, y donde a pesar de las medidas adoptadas, aun la posibilidad de desastre es real. Tomando como referencia a los estudios clásicos, realiza un breve análisis de la relación capital-trabajo. Brinda una mirada en un horizonte de proyección, donde junto a las imprescindibles medidas de mitigación y adaptación al cambio climático, se asumen otras asociadas a la emancipación humana. Igualmente, desde el análisis de algunos de los documentos normativos de los últimos años, se hace un acercamiento a la importancia de la educación como factor esencial para lograr detener este proceso. Se realiza una mirada crítica y cuestiona las distintas soluciones o propuestas relacionadas con el cambio climático. Al concluir se aprecia la necesidad de un cambio en este modelo de relaciones, fundamentalmente entre el ser humano y la naturaleza, para lograr la total emancipación. Además, se propone la educación como una de las herramientas más importantes como factor de cambio y donde el ser humano debe ser parte activa de este proceso de cambio.

\begin{abstract}
The present article analyses how critical thinking addresses the complex relationships between economic growth, as a model of prevailing social development and climate change. In this sense, climate change is presented as the most important global change facing humanity, and where despite the measures adopted, even the possibility of disaster is real. Taking as reference the classics, he makes a brief analysis of the Capital-work relationship. It provides a look at a projection horizon, where, together with the essential mitigation and adaptation measures to climate change, others associated with human emancipation are assumed. Likewise, from the analysis of some of the normative documents of recent years, an approach is made to the importance of education as an essential factor in stopping this process. It takes a critical look and questions the different solutions or proposals related to climate change. At the end, the need for a change in this model of relationships, fundamentally between the human being and nature, to achieve total emancipation is appreciated. In addition, education is proposed as one of the most important tools as a factor of change and where the human being must be an active part of this process of change.
\end{abstract}

\section{Palabras clave I Keywords}

Desarrollo social, crecimiento económico, cambio climático, pensamiento crítico, sujeto, modernidad.

Social development, economic growth, climate change, critical thought, subject, modernity.

Cómo citar: Rodríguez Pérez de Agreda, G.M., Cabalé Miranda, E., y Deroy Domínguez, D. (2019). El crecimiento económico como modelo de desarrollo social y su relación con el cambio climático. Retos Revista de Ciencias de la Administración y Economía, 9(18), 275-286. https://doi.org/ 10.17163/ret.n18.2019.06 


\section{Introducción}

El cambio climático (CC) impone a la sociedad un reto: o lo soluciona o la existencia de vida en nuestro planeta podría desaparecer. A pasar de las acciones nacionales e internacionales, la realidad actual no avizora un futuro promisorio: la temperatura global continúa en ascenso con la consiguiente degradación exponencial de los ecosistemas ¿Qué hacer? ¿Se trata de encontrar medidas de adaptación y mitigación más adecuada o debemos enfocar, además, nuestra mirada en otros lugares y procesos?

El presente trabajo aborda, desde el pensamiento crítico, el crecimiento económico como «modelo» de desarrollo social generalmente aceptado. Primero, siguiendo las pautas del pensamiento crítico, valora el condicionamiento histórico-social del crecimiento económico y de su traducción a «modelo ideal» del desarrollo social. De seguido se evalúan algunas de las consecuencias que este modelo acarrea en la realidad natural y social para luego, remontando sobre la propia época en que se impone, «cuestionar» las posibles soluciones al acuciante cambio global, en inextricable relación con el modelo de desarrollo social a seguir.

\section{El desarrollo como crecimiento económico}

La imagen más generalizada o "natural» que se tiene sobre el desarrollo de la sociedad es que este es una consecuencia del crecimiento económico. Se piensa, por ejemplo, que: "El crecimiento económico es importante, no por sí mismo sino por lo que permite hacer a un país y a las personas con los recursos que genera" (Programa de las Naciones Unidas para el Desarrollo (PNUD), 2014), idea que no refrenda la realidad práctica y por la que se reciben múltiples críticas (v. gr. Daly, 2012; Gómez \& Díaz, 2013, Rodríguez \& Cabalé, 2016; Kreimerman, 2017). Siguiendo estas detracciones, cabría señalar dos detalles elementales: en las sociedades pre-modernas el desarrollo no consistió en tal crecimiento, ni existe razón alguna para suponer que la sociedad post-capitalista tenga como criterio de progreso tal crecimiento; mientras que por otro lado, la idea de desarrollo como crecimiento "[...] cobró auge a partir del siglo XVIII, en el contexto de la lucha empeñada por la entonces joven burguesía contra el orden clerical-feudal..." (Acanda, 2002); es decir, la sociedad capitalista en su instauración y despliegue necesitó (y necesita hoy para su conservación) del crecimiento económico, pero la sociedad capitalista es solo un período en el devenir de la sociedad humana. La idea de progreso de la ilustración sirvió para la lucha en un contexto determinado, pero ese no es el contexto actual. Por ello cabe preguntarse ¿Qué es el crecimiento en sí y por qué se impone como "paradigma» de desarrollo social? Y, luego ¿Qué produce en la realidad tal crecimiento?

Para incursionar en el primer cuestionamiento propuesto, se debe valorar la relación social que vertebra la modernidad: la relación «Capital-Trabajo». Con frecuencia, cuando se analiza o estudia al capital, se le asume o define como un conjunto de equipos, materias primas, fábricas o dinero (Comisión Económica para América Latina y el Caribe [CEPAL], 2018) y se obvia o se desatiende lo esencial a él: su naturaleza necesariamente relacional. Por eso Marx (1973), para explicar en qué consiste el capital, comienza por enfatizar: "Una máquina de hilar algodón es una máquina para hilar algodón. Solo en determinadas condiciones se convierte en 
capital. Arracadas a esta condición, no tiene nada de capital...”. Es decir, el Capital, si bien lo pueden integrar mercancías, no son estas las que determinan su esencia, sino, la colocación de ellas en determinadas «condiciones específicas». Por ello, seguido de la aclaración, el filósofo alemán cuestiona y responde:

¿Cómo se convierte en capital una suma de mercancías, de valores de cambio? Por el hecho de que, en cuanto fuerza social independiente, es decir, en cuanto fuerza en poder de una parte de la sociedad, se conserva y aumenta por medio del intercambio con la fuerza de trabajo inmediata, viva (Marx, 1973, p. 164).

Primero, el capital no es un «conjunto de cosas», sino una «relación social específica»: Capital-trabajo, el trabajo asalariado presupone el Capital y el Capital presupone el trabajo asalariado, uno no pude existir sin el otro (Marx, 1973); segundo, en esa relación él es una fuerza social especial en manos de una clase social específica: la clase burguesa (la existencia de esta clase depende de esta fuerza y esta fuerza depende de la existencia de esa clase); tercero: como fuerza existe en un permanente "proceso de conservación y crecimiento" al entrar en relación con la fuerza de trabajo viva del obrero. Es por esta razón que más adelante Marx (op. cit.) precisa: "Un obrero en una fábrica algodonera ¿produce solamente tejidos de algodón? No, produce capital. Produce valores que sirven de nuevo para mandar sobre su trabajo y crear, por medio de este, nuevos valores" (Marx, 1973, p. 165).

Ahora ¿Qué resulta del proceso productivo capitalista? Resultan: «mercancías», un producto, en apariencias ${ }^{1}$, dirigido a la satisfacción de necesidades humanas; pero solo en "apariencia», habida cuenta, en realidad tal producto no está encaminado a ese fin, sino a la satisfacción de necesidades propias del Capital; un objeto que no procura la "satisfacción» de necesidades humanas, sino, por el contrario, "crearlas»; es un artículo dirigido no a satisfacer a un consumidor, sino a crearlo; un objeto que lo que pretende es realizar esa "conservación y crecimiento del capital» (Acanda, 2002). El crecimiento económico es la forma de existir y desarrollarse «el Capital», «no» la sociedad humana. La sociedad humana que necesita el crecimiento económico es la sociedad capitalista, no las que les precedieron, ni las que le sucedan.

Lo expuesto lleva a la respuesta de la segunda parte de la interrogante, el crecimiento económico se conforma como «el paradigma del desarrollo social» porque es pieza clave en la ideología hegemónica del capital (Gramsci, 1975), porque es un instrumento básico en la construcción de una imagen distorsionada y distorsionante de la sociedad, en virtud de la cual el orden capitalista se presenta como el «orden natural» de la sociedad humana (Acanda, 2002a), con lo cual algo «mejorable», pero nunca «sustituible».

Tomar el crecimiento económico como signo de desarrollo social no implica un «mero error epistemológico» o "posición teórica discutible» acerca del desarrollo de la sociedad, sino una construcción ideológica que, con fines de dominación ( $v$. gr. Boron, 2006), distorsiona la realidad social e implica serios problemas prácticos, a

1 Es importante señalar que la apariencia es algo consustancial a los fenómenos o proceso sociales de la modernidad, no es algo "casual» o una mera equivocación, artificio o error, sino algo «objetivo», propio del fenómeno, que permanentemente se oculta y se muestra como «no es» (Ramas, 2015). 
tal punto que en los países del llamado «socialismo real»-que lograron desmontar la producción capitalista- asumieron, precisamente, el crecimiento de la economía como sinónimo de desarrollo. Por ejemplo, afirman Odriozola y Triana (2015) acerca del proceso cubano actual: [...] "no es posible diseñar una estrategia de desarrollo sin tomar en cuenta la necesidad de alcanzar niveles sostenidos y sostenibles de crecimiento económico".

Se asume el crecimiento como mecanismo que «automáticamente» lleva al desarrollo social (Acanda, 2002a), algo que no ocurre en la práctica, pues se obvia un detalle de la realidad social actual: [...] "el nivel de desarrollo económico de un país, no es tanto una cuestión de producción, como de poder y posición” (Naredo, 2013, p. 170), no se tiene en cuenta que "la creencia en que un país desarrollado es un país muy productivo (...) eclipsa la realidad de que un país desarrollado es un país con poder suficiente para ejercer como atractor de capitales, recursos y población del resto del mundo" (Naredo, 2013, p. 171). La convicción en el automatismo de la economía y la ignorancia de esta realidad práctica "motivaron la crisis en la antigua Unión Soviética y, en general, de los países llamados «socialistas» que trataron de competir con el capitalismo en la carrera del desarrollo forzado de la «producción material" (Naredo, 2013, p. 175).

La idea o el argumento que se construyó alrededor del crecimiento económico fue que, al «crecer la riqueza» (cual recipiente que se vierte líquido continuamente) produce un «derrame» de ésta sobre resto de la sociedad, llevando así al bienestar y felicidad social (Daly, 2008, Arocena \& Sutz, 2013). Sin embargo, una simple mirada a la realidad social muestra una situación totalmente contraria, ya en el siglo XIX Engels exponía: “¿cuáles han sido las consecuencias de este acrecentamiento de la producción?, el aumento del trabajo agotador, una miseria creciente de las masas y un crac inmenso cada diez años" (Engels, 1974, p. 52). En esta misma línea, a finales del siglo XX el Programa de Naciones Unidas para el Desarrollo (PNUD) en su Índice de Desarrollo Humano advertía que "El crecimiento económico mundial casi nunca se filtra hacia abajo” (PNUD, 1992, p.1).

Elementalmente se puede asegurar que [...] "la obsesión por el crecimiento del PIB no garantiza una positiva evaluación del desarrollo social” (Sánchez \& Prada, 2015, p.223); es decir, por más que ha crecido la economía lo que ha derramado es pobreza, sin embargo lo importante no es tanto cuestionar «lo que no ha logrado como proyección», como los "desastres que realmente produce»

Ahora ¿Qué produce en la realidad «social»y «natural» tal crecimiento? El punto de inicio en este cuestionamiento es que: el modo de producir capitalista se desentiende de las consecuencias «sociales» $\mathrm{y}$ «naturales» de sus actos (Engels, 1974a; Kreimerman, 2017).

En lo social, el crecimiento del Capital acarrea dos consecuencias esenciales a saber: Primero, aumento de la dominación sobre el ser humano a través de su conversión en «obrero», pues "Al crecer el capital, crece la masa del trabajo asalariado, crece el número de obreros asalariados; en una palabra, la dominación del capital se extiende a una masa mayor de individuos" (Marx, 1973, p. 166), o lo que es lo mismo, con el crecimiento económico el Capital logra un aumento del poder en «intensidad» y en «extensión» sobre resto de la sociedad. Implica una polarización de la riqueza-poder 
y la pobreza-subordinación, donde la primera se acumula en una parte cada vez más pequeña de la sociedad y la otra se extiende a una parte cada vez mayor de esa sociedad.

Segundo; la dominación del ser humano a través de su conversión en «consumidor»: un individuo que pierde la capacidad de conformar y satisfacer sus necesidades por sí mismo y queda subordinado o directamente sometido a los designios del mercado. Un ser humano en al cual "no solo sus necesidades, sino también su modo de satisfacerlas y el modo de representárselas, tienen que existir como función del consumo no de cualquier tipo de objetos o «cosas», sino de un objeto muy específico: la mercancía" (Acanda, 2002, s/p).

Las desbastadoras consecuencia que el crecimiento económico del capital produce (v. gr. Morejón, 2015; Kreimerman, 2017) se deben, entre otras, a una mutación que acarreó su aparición con la modernidad, él [...] "implicó la transformación en mercancías de tres bienes fundamentales para la sociedad: la fuerza de trabajo, la tierra y la moneda. Sus consecuencias han sido y continúan siendo dramáticas para la sociedad" (Acanda, 2002a, p. 79)

\section{Crecimiento económico y sus consecuencias en la naturaleza}

Las apreciaciones y advertencias sobre las desbastadoras consecuencias naturales del crecimiento no son recientes, e incluso tienen antecedentes en el propio siglo XVIII en una corriente de economistas denominados hoy como «fisiócratas» (Gómez \& Díaz, 2013; Naredo, 2004 y 2013; Pérez, 2016). En el siglo XIX, varios investigadores de las ciencias naturales (físicos, biólogos, químicos...), advirtieron a los economistas de entonces acerca de las graves consecuencias que traería a la economía los acontecimientos naturales que acarreaba el crecimiento; "sus teorías, sin embargo, no fueron consideradas seriamente por la economía convencional” (Gómez \& Díaz, 2013, p.9).

En esa misma época, Engels advirtió de las graves consecuencias que traerían para los suelos, los métodos para optimizar el cultivo del café en zonas montañosas (Engels, 1974a). A mediados de ese siglo, desde las ciencias económicas, Thomas Robert Malthus, David Ricardo y John Stuart Mill alertaron que, de seguir el ritmo de crecimiento, se podría llegar a un «estado estacionario» debido al agotamiento de los recursos naturales (Naredo, 2004; Gómez \& Díaz, 2013), una alerta que aún hoy se conserva vigente, pero no precisamente por un "problema económico» (de no poder producir por escases de materias primas), sino, por algo mucho más grave: «el peligro de la subsistencia de la propia vida en la tierra», y no tanto por la desaparición de recursos, sino por la generación y deposición incontrolable de residuos y la destrucción de ecosistemas (Naredo, 2004; Garea, 2014) promotores del CC, calificado como el más gravitante de los cambios globales (Cuba, 2017) sobre el cual Ban Ki-moon sentenció: "El cambio climático es el problema que define nuestra era. Define nuestro presente. Nuestra respuesta definirá nuestro futuro. Para navegar a través de esta tormenta requerimos de todas las manos sobre cubierta" (Ban Ki-moon citado en Aguilar, Granat, \& Owren, 2017, p.19).

Una mirada a este complejo proceso debe comenzar por precisar ¿Qué debe entenderse por CC? Ya que, con frecuencia, se asocia a eventos meteorológico que ocurren en un momento determinado, cuando no es propiamente así. Cabe señalar 
que una cosa es el «tiempo atmosférico»y otra cosa es el «clima», el primero se entiende como:

[...] el estado físico de la atmósfera caracterizado por el conjunto de los valores instantáneos de las variables atmosféricas (temperatura, presión, humedad, velocidad del viento, otras) en un lugar dado e instante determinado, o por los cambios y valores medios de dichas variables durante un plazo de tiempo relativamente corto (Garea, 2014, p.19).

El tiempo atmosférico es, por esencia, cambiante, mientras que el «clima» se puede conceptualizar como:

[...] el estado promedio del tiempo y, más rigurosamente, como una descripción estadística del tiempo atmosférico en términos de los valores medios y de la variabilidad de las magnitudes correspondientes durante períodos que pueden abarcar desde meses hasta miles o millones de años (Intergovernmental Panel on Climate Change [IPCC], 2014, p. 130).

El clima es, entonces, algo relativamente estable o permanente en el tiempo y en el lugar, por ello se pueden identificar climas: «húmedos», «secos», "fríos», «templados», etc. El clima en la tierra está determinado por la energía que recibe del sol (Garea, 2014) y el hecho que sea relativamente estable por largos períodos de tiempo, no quiere decir inmodificable, él ha variado cíclicamente a lo largo de la historia, debido "... a procesos internos naturales o a forzamientos externos tales como modulaciones de los ciclos solares, erupciones volcánicas...” (IPCC, 2014, p. 129).

Esta mutabilidad del clima en su devenir se denomina CC, el cual es conceptualizado por el IPCC (op. cit., p.129) como una "variación del estado del clima identificable (p. ej., mediante pruebas estadísticas) en las variaciones del valor medio o en la variabilidad de sus propiedades, que persiste durante largos períodos de tiempo, generalmente decenios o períodos más largos". Este proceso puede ocurrir por cambios naturales o antropógenos, por ello la Convención Marco de las Naciones Unidas sobre el Cambio Climático (CMNUCC), se centra, al conceptualizarlo, como un "cambio de clima atribuido directa o indirectamente a la actividad humana que altera la composición de la atmósfera global y que se suma a la variabilidad natural del clima observada durante períodos de tiempo comparables" (CMNUCC citada en IPCC, 2014, p. 129).

El clima de la tierra es consecuencia, en lo fundamental, del proceso de transformación de la energía solar en terrestre. Este proceso resulta de la interacción de la radiación solar con la atmosfera, la superficie de la tierra y los océanos. Por esa razón, son determinantes en tal proceso la composición y características de la atmósfera y las particularidades de la superficie terrestre y la de los océanos (Garea, 2014). Ellos son responsables del denominado balance radiactivo y ciclo hidrológico (Garea, 2014)

La vida en la tierra se debe, en gran medida, a su atmósfera, a su composición y características que permiten que la energía solar, reflejada por la superficie terrestre, no escape al espacio y se irradie nuevamente hacia ella, provocando lo que se conoce como «efecto invernadero». De este proceso son responsables un conjunto de gases denominados, entonces: "gases de efecto invernadero (GEI)». Si estos gases no formaran parte de la atmósfera la temperatura media de la Tierra fuera del orden 
de $-18^{\circ}$ C" (Garea, 2014). Los principales GEI, además del vapor de agua (parte del importante ciclo hidrológico), son: el dióxido de carbono, el ozono, el metano y el óxido nitroso. La presencia y concentración de estos gases en la atmosfera depende de la correlación entre la emisión de estos por fuente naturales o humanas y de su absorción por sumideros.

Debido, por una parte, a la actividad productiva y, por otra, a la destrucción de los sumideros (tala indiscriminada de bosques, etc.), motivado por la necesidad de lograr el «crecimiento económico», han aumentado progresivamente la concentración en la atmosfera del: «dióxido de carbono»² (consecuencia de la combustión del carbón, petróleo y gas natural), «metano y el óxido nitroso» (debido a la agricultura, la descomposición de la materia orgánica, lo vertederos y a los cambios en el uso de la tierra) (Garea, 2014; Delgado et al., 2018). Además, debido a la producción humana, se suman a esa concentración los «clorofluorocarbonos» (CFC), los «hidroclorofluorocarbonos» (HFC) y los «hidrocarburos perfluorados» (PFC), sustancias que no existen por sí solas en la naturaleza y que tienen un alto poder de calentamiento en la atmósfera (Camacho et al., 2018; Barrientos, Méndez, \& Welsh, 2019). Todos ellos han llevado a un aumento progresivo de la temperatura en la tierra con el consecuente CC.

Los problemas asociados al CC no solo estriban en los cambios en sí, sino, además, en la velocidad con que estos ocurren, lo cual impide o anula la capacidad de los ecosistemas a acomodarse a tales cambios y "con esto devienen procesos de degradación ecológica irreversibles” (Garea, 2014, p. 24). De allí que el CC sea el proceso ambiental más acuciante y grave al que se enfrenta el hombre, pues él produce un efecto de cascada de depauperación sobre las aguas, los suelos, los bosques y en general, sobre todos los elementos del medio natural en que descansa la vida y:

[...] si ocurren cambios en el medio ambiente mundial como alteraciones en el clima, en la productividad del suelo, en los recursos oceánicos o de agua dulce, en la química de la atmósfera o en la ecología de los sistemas, se podría alterar la capacidad del Planeta para sustentar la vida (Garea, 2014, p. 13).

Por otra parte, si bien el cambio climático es de naturaleza global, presenta efectos regionales que difieren de manera considerable (Aguilar et al., 2017), además lo hará de manera diferenciada en las personas, "Los pobres, (...) se verán afectados de manera desproporcionada." (Global Gender and Climate Alliance [GGCA], 2009, p. 87). De hecho, el CC podría ahondar la ya profunda brecha entre ricos y pobres. Entonces, el hecho que el CC sea un proceso global, no implica que sus impactos sean similares en todos los lugares y personas del planeta, ello dependerá de la singularidad del territorio. De allí que, si bien requiere medidas globales, en esa misma medida necesita soluciones esencialmente locales, dada la singularidad de sus diversos impactos.

Las acciones que se proponen frente al CC son acciones de «mitigación», encaminadas a controlar o eliminar las causas que generan el cambio y acciones de «adaptación» enfocadas en desarrollar la capacidad de adaptación a las nuevas con-

2 "La concentración global de dióxido de carbono (CO2) en la atmósfera ha aumentado de aproximadamente 280 partes por millón (ppm) a finales del siglo XVIII hasta más de 400 ppm de CO2 en 2015” (Vergara, Fenhann \& Schletz, 2016, p1). 
diciones que él impone. Ahora, si bien metodológicamente se pueden abordar por separado las acciones mitigación y de adaptación, orgánicamente deben funcionar como una sola, es decir, deben ser una integridad, pues mitigar la emisión de gases, no impide los efectos que el CC hoy impone a la sociedad. Por otra parte, dedicar los esfuerzos solo en adaptarnos, deja indemnes las causas que nos llevan a ello, deja en pleno desarrollo la cascada de destrucción que impone el CC; por ello se debe llevar un accionar conjunto.

Sin embargo, a pesar de definirse estas acciones frente al CC, a pesar de existir todo un programa internacional para enfrentar este cambio, la depauperación continúa, pues parece que mantener el objetivo de $2{ }^{\circ} \mathrm{C}$ es cada vez más inalcanzable (Vergara et al., 2016). El deterioro continúa su marcha y ello se debe a que la causa que lo produce permanece allí: el modo en que se produce y reproduce la sociedad continúa de la misma forma, la idea que se tiene de «desarrollo» (con independencia del adjetivo "sostenible») como "crecimiento económico» continúa siendo la misma, con lo cual el imperio del mercado continúa inalterable ¿Qué hacer?

\section{Miradas críticas a las soluciones}

En este punto el problema no consiste tanto en encontrar la respuesta a la interrogante, como en cuestionar las soluciones planteadas. Los problemas que presenta el CC no deben verse al margen de los problemas que engendra el modelo "hegemónico» de desarrollo social. Por ello no se deben tratar solas las acciones de mitigación y adaptación, sino cuestionar, además, el propio modelo de desarrollo imperante, al cual generalmente solo se le reprocha una «mala distribución de la riqueza».

Por ejemplo, el PNUD en su Informe de Desarrollo Humano: 1992 en la caratula del mismo grafica que "El 20\% más rico de la población mundial recibe el 82.7\% de los ingresos totales del mundo, mientras que el $20 \%$ más pobre tan sólo recibe el $1.4 \%$ ". Sin embargo, luego en el análisis del informe identifica al crecimiento económico como "un medio importante" para alcanzar el desarrollo humano (PNUD, 1992), sin tomar en cuenta que, justamente, esa mala distribución de la riqueza que refieren es una «consecuencia obligada» del crecimiento económico, ni más ni menos; con lo cual la solución del problema no está en colocar al Estado cual «instrumento de redistribución» de la riqueza.

La redistribución de la riqueza a través del Estado si bien, de alguna manera, "amortigua» las pésimas condiciones de existencia de las clases desposeídas, no deja de ser una manera de conservar la dominación y la asimetría social, en razón a que serán los desposeídos, los que reciban estas ayudas del Estado para satisfacer sus necesidades (fundamentalmente las perentorias), en consecuencia, no serán libres de elegir qué o cómo satisfacer sus necesidades, en razón a que esta posibilidad de satisfacción le está vetada por la realidad y le proviene, entonces, de un organismo que le mediatiza en su satisfacción, no es de sí mismo. En este punto quedan intactas las grandísimas diferencias de necesidades y satisfacciones entre el desposeído y el capitalista.

El problema del Capital es la creciente polarización de la riqueza-poder y pobreza-subordinación que engendra, algo que no puede solucionar el Estado paliando, elementalmente, la enorme depauperación social que este proceso, de manera 
"natural”, genera. La redistribución estatal atenúa el estado de cosas, pero siempre de manera limitada, parcial, pues, como advierte Marx (1973), las necesidades y goces sociales son siempre relativas, son objetivamente históricas concretas, que el capital coloca en un proceso de incremento exponencial, en consecuencia, el desposeído, el asalariado jamás podrá acceder a las necesidades y goces del capitalista. Por esa elemental razón como ha demostrado la historia más reciente, al totalitarismo del mercado no se le puede enfrentar con el totalitarismo del Estado (Acanda, 2002).

Tal vez el cuestionamiento comience por no asumir la realidad social con simpleza y negarla en bloque como: «equivocada», «desafortunada» o «destructora», sino, de superarla críticamente para lo cual se requiere, entonces, remontarse sobre la época histórica en que vivimos: «la modernidad»(Giddens, 1998, Acanda, 2002a) y encontrar en sus contradicciones el camino de salida.

La irrupción del modo de producción capitalista en ese período histórico generó cambios radicales en las relaciones sociales, en razón a que, por primera vez en la historia de la humanidad, la producción de las riquezas sociales se fundó en la relación directa de hombres libres. Estas relaciones básicas de la sociedad necesariamente transformaron todas las restantes relaciones entre los hombres (Acanda, 2002a). La ideología del Capital que se forma sobre estas bases: el Liberalismo, produjo un cambio sustancial en la idea y representación de las relaciones del hombre con la sociedad, mostrando un nuevo paradigma de lo social, pues consistía en llegar a pensar todo el problema de la existencia social a partir del individuo (Acanda, 2002a). Apareció, así, la idea del hombre como «sujeto» de su propia vida: un individuo, entre otras, con capacidad para definir sus propios objetivos y modos de alcanzarlos, capaz de construirse a sí mismo en la medida que transforma la realidad que le rodea (D’Angelo, 2001; Acanda, 2008; Hinkelammert, 2010).

Sin embargo, esta vocación de liberación, de pleno desarrollo de la subjetividad humana no se puede desplegar en medio de una sociedad dominadora como la sociedad burguesa. La necesidad de producir más y más baratas mercancías y obtener más ganancias, hizo aparecer la racionalidad instrumental, una racionalidad que puede ser sintetizada: en el desarrollo de la ciencia y el dominio de la naturaleza para a través de ella dominar al hombre, como obrero o como consumidor (Acanda, 2002; Hinkelammert, 2010a).

Pero, aun cuando la modernidad se traiciona a sí misma, no puede ser apartada, sin más, como errónea, sino debe ser superada críticamente, para lo cual primero se debe rescatar el propósito de alcanzar el pleno desarrollo de la subjetividad humana, lograr la conformación del ser humano en «sujeto» de su propia vida, lo cual implica despojar el proceso de conformación de la subjetividad humana de la redes de dominación que la racionalidad instrumental pretende extender sobre ella (Acanda, 2002) y; segundo, lograr el desarrollo de una racionalidad humana liberadora, habida cuenta, es imposible lograr la emancipación humana sin conocer y dominar la realidad que nos rodea (Cabalé \& Rodríguez, 2016), pero ese proceso de conocer y dominar no puede estar dirigido a dominar y someter al propio hombre.

Habría que reconocer que el propósito de aquel entonces, como el que hoy nos ocupa, está estrechamente relacionado, entre otras, con la Educación, justamente por ello en la Agenda 2030, donde se señala como objetivo número 13 "Adoptar medidas 
urgentes para combatir el cambio climático y sus efectos", para conseguir este, prescribe en su meta 13.3 "Mejorar la educación, la sensibilización y la capacidad humana e institucional respecto de la mitigación del cambio climático, la adaptación a él, la reducción de sus efectos y la alerta temprana" (CEPAL, 2016, p.33). Sin embargo, no se trata solo de educar para la mitigación y adaptación al CC, sino de ir más allá y buscar la superación crítica de estas contradicciones y aporías modernas. No se trata de «salvar a la humanidad» de una catástrofe, sino que «la humanidad se salve» de ella y eso implica que sea el propio ser humano el artífice de su meta, un ser humano «sujeto de su propio destino».

Esta educación para el cambio puede servirse de determinados presupuestos existente en la región latinoamericana: por una parte, recientes encuestas de opinión destacan que América Latina es una de las regiones donde existe mayor preocupación por el CC y refieren mayor sensación de afectaciones por el calentamiento global, por otra, "refleja una tensión permanente entre proyectos de vida autónomos (...) y proyectos más instrumentales" (PNUD, 2016).

\section{Conclusiones}

El CC, uno de los más gravitante procesos globales al cual se enfrenta la humanidad, es consecuencia, en medida importante, del modelo de desarrollo centrado en el crecimiento económico, que implica, por necesidad, un «consumo irracional» de recursos naturales y «contaminación exponencial» del ambiente, para la producción de «mercancías», objeto cuyo propósito oculto, pero real, es la conservación y aumento de la dominación de una parte cada vez menor de la sociedad sobre una parte cada vez mayor de esta; en consecuencia, la superación de este proceso de cambio global, en buena medida, implica un cambio en el modelo de desarrollo social, no centrado en la producción de "cosas», sino en la emancipación humana, en la sustitución de relaciones intersubjetivas de dominación, por relaciones intersubjetivas de emancipación, que implica, necesariamente, un cambio en la relaciones entre los seres humanos y de esto con la naturaleza de la cual son parte.

La capacidad adaptativa del ser humano -imprescindible frente a los retos que imponen el CC- no se desarrolla en un medio de subordinación, de dominación, o lo que es lo mismo, no es posible en una relación donde unos pocos son sujetos de sus actos y otros muchos objetos de esos actos, esa capacidad adaptativa imprescindible es parte de relaciones entre humanos sujetos de sus propias vidas.

La singularidad de los impactos del CC en lugares y personas, apunta, al menos, dos perspectivas necesarias: las soluciones no pueden ser únicas y generales sino singulares y locales, en justa correspondencia con esto, el desarrollo debe ser local y endógeno, por su parte los impactos diferenciados en las personas acarrearan una profundización en las desigualdades entre ricos y pobres, de allí que se necesite un paradigma de desarrollo social que se sitúe en las coordenadas la verdadera emancipación humana. 


\section{Referencias}

Acanda, J. L. (2002). ¿Qué significa ser progresista en materia de pensamiento? Recuperado el 22 de julio de 2015, de http://biblioteca.filosofia.cu/php/export.php?format=htm\&id=37\&view $=1$

Acanda, J. L. (2002a). Sociedad Civil y Hegemonía. La Habana, Cuba: Centro de Investigaciones y Desarrollo de la Cultura cubana Juan Marinello.

Acanda, J. L. (2008). La problemática del sujeto y los desafíos para la teoría de la educación, Rebelión, 1 de julio. Recuperado de https://bit.ly/2YFglwd

Aguilar, L., Granat, M., \& Owren, C. (2017). Las Raíces del futuro. Situación actual y progreso en género y cambio climático. Las raíces del futuro: Situación actual y progreso en género y cambio climático. Washington DC, Estados Unidos: UICN y GGCA.

Arocena, R., \& Sutz, J. (2013). Innovación y democratización del conocimiento como contribución al desarrollo inclusivo. En G. Dutrénit \& J. Sutz (Eds.), Sistemas de innovación para un desarrollo inclusivo. La experiencia latinoamericana (pp. 19-34). Ciudad México: Foro Consultivo Científico y Tecnológico.

Barrientos-Cruz, A. R., Méndez-Salazar, M. A., \& Welsh-Rodríguez, C. M. (2019). Análisis inter-temporal de la contaminación por gases de efecto invernadero: Avances teóricos y perspectivas para México. DIGITAL CIENCIA@UAQRO, 12(1), 60-68. https://bit.ly/2KbI0kz

Boron, A. A. (2006). Diálogo sobre el poder, el Estado y la Revolución. La Habana, Cuba: Centro de Investigación y Desarrollo de la Cultura cubana Juan Marinello.

Cabalé, E., \& Rodríguez, G. (2016). El desarrollo a propósito del pensamiento de Rodolfo Stavenhagen. Estudios del Desarrollo Social: Cuba y América Latina, 4(3), 82-96.

Camacho, C., Marmolejo, Y., Otazo, E. M., \& Romo, C. (2018). Emisiones de GEI del corredor industrial Apaxco-Tula. Publicación Semestral Pädi 7(13), 12-16. https://doi.org/10.29057/icbi. v7i13.3443

Comisión Económica para América Latina y el Caribe (CEPAL). (2016). Agenda 2030 y los Objetivos de Desarrollo Sostenible: una oportunidad para América Latina y el Caribe. Recuperado de https://bit. ly/2zpSrfb

Comisión Económica para América Latina y el Caribe (CEPAL). (2018). Estudio Económico de América Latina y el Caribe, 2018. Santiago, Chile: Naciones Unidas. Recuperado de https://bit. ly/2SQeWAS

República de Cuba. (2017). Segunda Comunicación Nacional a la Convención Marco de las Naciones Unidas sobre Cambio Climático. La Habana, Cuba: Editorial AMA. Recuperado de https://bit. ly/2YEoMIe

D’Angelo, O. (2001). Sociedad y educación para el desarrollo humano. La Habana, Cuba: Pueblo y Educación.

Daly, H. (2008). Desarrollo Sustentable: definiciones, principios, políticas. Aportes, (7), 3-26.

Daly, H. (2012). Ocho falacias sobre el Crecimiento, Otro desarrollo, 3 de mayo de 2016. Recuperado de https://bit.ly/2OzB5FW

Delgado, J., Yépez, E. A., Paz, F., Ángeles, G., Aguirre, C., Alvarado, M. S., et al. (2018). Base de datos de flujos verticales de dióxido de carbono en ecosistemas terrestres y costeros en México. Elementos para políticas públicas, 2(2), pp. 93-108. Recuperado de https://bit.ly/2YDhlVO

Engels, F. (1974). Introducción a la Dialéctica de la Naturaleza. En Obras Escogidas de C. Marx y F. Engels (pp. 39-56). Moscú, Rusia: Progreso.

Engels, F. (1974a). El papel del trabajo en la trasformación del mono en hombre. En Obras Escogidas de C. Marx y F. Engels (pp. 66-79). Moscú, Rusia: Progreso.

Garea, B. (Coord.). (2014). Cambio Climático y Desarrollo Sostenible. Bases Conceptuales para la Educación en Cuba. La Habana, Cuba: Editorial Educación Cubana.

Giddens, A. (1998). Modernidad e identidad del yo. El yo y la sociedad en la época contemporánea. Barcelona, España: Ediciones Península. Recuperado de https://bit.ly/2Htqhoa

Global Gender and Climate Alliance (GGCA). (2009). Manual de capacitación en género y cambio climático. San José, Costa Rica: Masterlitho S.A. 
Gómez, C., \& Díaz, J. A. (2013). Origen del concepto de desarrollo sostenible. En Referencias para un análisis del desarrollo sostenible (pp. 7-16). Alcalá de Henares, España: Universidad de Alcalá.

Gramsci, A. (1975). Cuadernos de la Cárcel. Edición crítica del Instituto Gramsci (2da Edición ed., Vol. Tomo I). (A. M. Palos, Trad.) México: Ediciones Era.

Hinkelammert, F. J. (2010). Retorno del sujeto humano reprimido frente a la estrategia de globalización. En Ecología política y Educación Popular ambiental (pp. 21-25). La Habana, Cuba: Editorial Caminos.

Hinkelammert, F. J. (2010a). La irracionalidad de lo racionalizado. Comentarios metodológicos sobre la racionalidad instrumental y su totalización. En Ecología política y Educación Popular ambiental (pp. 75-77). La Habana, Cuba: Editorial Caminos.

Intergovernmental Panel on Climate Change (IPCC). (2014). Anexo II: Glosario. En K. J. Mach, S. Planton, \& C. von Stechow (Eds.), Cambio climático 2014: Informe de síntesis. Contribución de los Grupos de trabajo I, II y III al Quinto Informe de Evaluación del Grupo Intergubernamental de Expertos sobre el Cambio Climático [Equipo principal de redacción, R.K. Pachauri y L.A. Meyer] (pp. 127-141). Ginebra, Suiza: IPCC. Recuperado de https://bit.ly/2ZrW7Hy

Kreimerman, R. (2017). Contexto económico en América Latina. Perspectivas y tendencias para la transformación social-ecológica, Fundación Friedrich-Ebert-Stiftung (FES Transformación), diciembre. Recuperado de https://bit.ly/3194cRU [Fecha de consulta: 10 de junio de 2019]

Marx, C. (1973). Trabajo asalariado y capital. En Obras Escogidas de C. Marx y F. Engels (pp. 145-178). Moscú, Rusia: Progreso.

Morejón, A. (2015). Significación del capital en la crisis ambiental. Breve abordaje teórico desde el debate filosófico. Revista cubana de Ciencias Sociales, (45), 83-96.

Naredo, J. M. (2004). Sobre el origen, el uso y el contenido del término sostenible. En Cuadernos de Investigación Urbanística (pp. 7-18). Madrid, España: Instituto Juan de Herrera.

Naredo, J. M. (2013). Perspectivas de sostenibilidad en Cuba. En A. Gómez Sal, \& C. Gómez Gutiérrez (Coords)., Reflexiones sobre el desarrollo sostenible en Cuba: Una mirada desde el mundo académico (pp. 169-179). Alcalá de Henares, España: Universidad de Alcalá de Henares.

Odriozola, S., \& Triana, J. (2015). Estrategia de desarrollo y crecimiento económico en Cuba: dos caras de una misma moneda. Economía y Desarrollo, 153 (Número Especial),. 14-29. Recuperado de https://bit.ly/2Yiu80I

Pérez, I. E. (2016). Las teorías del crecimiento económico: notas críticas para incursionar en un debate inconcluso. LAJED, (25), 73-125.

Programa de las Naciones Unidas para el Desarrollo (PNUD). (1992). Desarrollo Humano: Informe 1992. Santafé de Bogotá, Colombia: Tercer Mundo Editores. Recuperado de https://bit. ly/2LVM5eF

Programa de las Naciones Unidas para el Desarrollo (PNUD). (2014). Informe sobre Desarrollo Humano 2014. Sostener el Progreso Humano: Reducir vulnerabilidades y construir resiliencia. New York, Estados Unidos: PNUD. Recuperado de https://bit.ly/2ZlhbQ5

Programa de las Naciones Unidas para el Desarrollo (PNUD). (2016). Informe Regional sobre Desarrollo Humano para América Latina y el Caribe. Progreso multidimensional: bienestar más allá ingreso. New York, Estados Unidos: PNUD. Recuperado de https://bit.ly/2Xh6u3l

Ramas, C. (2015). Hacia una teoría de la apariencia: fetichismo y mistificación en la crítica de la economía política de Marx (Tesis Doctoral). Facultad de Filosofía Universidad Complutense de Madrid, España. Recuperado de: https://eprints.ucm.es/34214/1/T36659.pdf

Rodríguez, G., \& Cabalé, E. (2016). Desarrollo sostenible y crecimiento económico ¿Conceptos compatibles? Estudios del Desarrollo Social: Cuba y América Latina, 4(4), 131-144.

Sánchez, P., \& Prada, A. (2015). Del concepto de crecimiento económico al de desarrollo de las naciones: una aplicación a la Unión Europea. Revista de Economía Mundial, (40), 221-251.

Vergara, W., Fenhann, J. V., \& Schletz, M. C. (2016). Carbono cero. América Latina una vía para la descarbonización neta de la economía regional para mediados de este siglo: documento de visión. UNEP DTU Partnership. Recuperado de https://bit.ly/1ROpkGP 